\title{
Inverse degrees and the Jacobian conjecture
}

\author{
Harm Derksen \\ University of Nijmegen \\ Department of Mathematics \\ Toernooiveld \\ 6525 ED Nijmegen \\ The Netherlands \\ E-mail: hderksen@sci.kun.nl
}

\begin{abstract}
Bass, Connell and Wright proved that if the Jacobian Conjecture is true, then there exists a number $C(d)$ such that for every $k$-algebra $A$ and every invertible polynomial map $F: A^{n} \rightarrow A^{n}$ with $\operatorname{det}(J(F))=1$ the degree of $F^{-1}$ is bounded by $C(d)$. A year later Bass proved the converse. In this paper we give a short proof of this last result.
\end{abstract}

In this paper $k$ is a field of characteristic 0 . If $F=\left(F_{1}, \ldots, F_{n}\right): k^{n} \rightarrow k^{n}$ is a polynomial map, then we define the degree of $F$ as the maximum degree of the polynomials $F_{1}, \ldots, F_{n}$. This paper is about the equivalence of the following two conjectures:

Conjecture $1 \mathrm{JC}(n):$ If $F: k^{n} \rightarrow k^{n}$ is a polynomial map with $\operatorname{det}(J(F))=1$ then $F$ is invertible, e.g. there exists a polynomial map $G: k^{n} \rightarrow k^{n}$ such that $G \circ F=F \circ G=\mathrm{id}$.

This conjecture is known as the Jacobian conjecture (c.f, $[3,4,6]$ ), and it was first formulated by $\mathrm{O} . \mathrm{H}$. Keller in [5].

Conjecture $2 \mathrm{BI}(n)$ : For every finite dimensional $k$-algebra $A$ the following holds:

If $F: A^{n} \rightarrow A^{n}$ is an invertible polynomial map such that $\operatorname{det}(J(F))=1$, then the degree of $F^{-1}$ is bounded by a constant $C(d)$, depending only on $d$ (and $n$ ), and not on the choice of $A$.

If $A$ is a field, then one can take $C(d)=d^{n-1}$. This result is due to Gabber (c.f. [2, Ch. I, Cor. (1.4). ). if $A$ is a reduced ring, then it is easy to reduce it to the case that $A$ is a ficld. For a reduced ring $A$ we can also take $C(d)=d^{n-1}$ as the upper bound. If $A$ is allowed to have nilpotent elements then it is not known whether there can be given an upper bound for the degree of the inverse. In [2, Ch. I, Prop. (1.2).] Bass, Connell and Wright proved that $\mathrm{JC}(n)$ implies $\mathrm{BI}(n)$. Bass proved in [1] the converse. We will now give a short proof of this implication:

Theorem 1 If the conjecture $\mathrm{BI}(n)$ is true then the jacobian conjecture $\mathrm{JC}(n)$ is true.

1991 Mathematics Subject Classification 14E09. 
Proof: Assume $\mathrm{BI}(n)$ holds and assume that $F: k^{n} \rightarrow k^{n}$ is a polynomial map satisfying $\operatorname{det}(J(F))=1$. Without loss of generality we may assume that $F(0)=0$. Now $F$ is locally invertible in the neigbourhood of 0 , e.g. there exist $G_{1}, G_{2}, \ldots, G_{n} \in k\left[\left[X_{1}, \ldots, X_{n}\right]\right]$ such that $F_{i}\left(G_{1}, \ldots, G_{n}\right)=X_{i}$ for all $i$. Write

$$
F=F_{(1)}+F_{(2)}+\ldots+F_{(d)} \text { and } G=G_{(1)}+G_{(2)}+G_{(3)}+\ldots
$$

where $F_{(i)}$ and $G_{(i)}$ are homogeneous of degree $d$ for all $i$. Let us define $\hat{F}: k[t]^{n} \rightarrow k[t]^{n}$ by

$$
\widehat{F}=t^{-1} F(t X)=F_{(1)}+t F_{(2)}+\ldots+t^{d-1} F_{(d)}
$$

and likewise define

$$
\widehat{G}=t^{-1} G(t X)=G_{(1)}+t G_{(2)}+t^{2} G_{(3)}+\ldots
$$

Since $J(\hat{F})=J\left(t^{-1} F(t X)\right)=J(F)(t X)$, we have $\operatorname{det}(J(\hat{F}))=\operatorname{det}(J(F))(t X)=1$. Now $\hat{G}$ is the formal inverse of $\hat{F}$ because

$$
\hat{F} \circ \hat{G}=t^{-1} F\left(t t^{-1} G(t X)\right)=t^{-1} F(G(t X))=t^{-1} t X=X
$$

Choose $l>C(d)$ arbitrary. We now calculate modulo $t^{l}$. Define $\bar{F}:\left(k[t] /\left(t^{l}\right)\right)^{n} \rightarrow\left(k[t] /\left(t^{l}\right)\right)^{n}$ and $\bar{G}$ to be $\widehat{F}$ respectively $\hat{G}$ modulo $t^{l}$. Again we $\operatorname{get} \operatorname{det}(J(\bar{F}))=1$ and $\bar{F} \circ \bar{G}=X$. So $\bar{G}$ is the inverse of $\bar{F}$, hence the degree of $\bar{G}$ is bounded by $C(d)$. Since

$$
\bar{G}=G_{(1)}+t G_{(2)}+\ldots+t^{l-1} G_{(l)}
$$

it follows that $t^{l-1} G_{(l)}=0$. The fact that $t^{l-1} \neq 0$ (in $k[t] /\left(t^{l}\right)$ ) forces $G_{(l)}=0$. We can conclude that $G_{(l)}=0$ for alle $l>C(d)$. So $G$ is also a polynomial map and this proves that $F$ is invertible.

\section{References}

[1] H. Bass, The Jacobian conjecture and inverse degrees, Arithmetic and geometry, Prog. Math. 36, 65-75 (1983).

[2] H. Bass, H. Connell and D. Wright, The Jacobian conjecture: reduction of degree, and formal expansions of the inverse, Bull. Amer. Math. Soc. (1982).

[3] L. Drużkowski, The jacobian conjecture, preprint 492, Inst. of Math., Polish Academy of Sciences, Warsaw, 1991.

[4] A. van den Essen, Polynomial maps and the Jacobian conjecture, Computational aspects of Lie group representations and related topics, of the 1990 computational algebra seminar, in C.W.I. Tract. 84 (1991), 29-24.

[5] O.H. Keller, Ganze Cremona-Transformationen, Monats. Math. Physik 47 (1939), 299-306.

[6] K. Rusek, Polynomial automorphisms (preprint 1989). 